\title{
Inhibition of RhoA-Subfamily GTPases Suppresses Schwann Cell Proliferation Through Regulating AKT Pathway Rather Than ROCK Pathway
}

\author{
Dandan Tan 1,2, Jinkun Wen 1,2, Lixia Li 1,2, Xianghai Wang 1,2, Changhui Qian ${ }^{1,2,3}$, \\ Mengjie Pan ${ }^{1,2}$, Muhua Lai ${ }^{1,2}$, Junyao Deng ${ }^{1,2}$, Xiaofang $\mathrm{Hu}^{1,2}$, Haowen Zhang ${ }^{1,2}$ \\ and Jiasong Guo ${ }^{1,2,4 *}$ \\ ${ }^{1}$ Guangdong Provincial Key Laboratory of Construction and Detection in Tissue Engineering, Southern Medical University, \\ Guangzhou, China, ${ }^{2}$ Department of Histology and Embryology, Southern Medical University, Guangzhou, China, \\ ${ }^{3}$ Department of Histology and Embryology, Fujian University of Traditional Chinese Medicine, Fuzhou, China, ${ }^{4}$ Key Laboratory \\ of Mental Health of the Ministry of Education, Southern Medical University, Guangzhou, China
}

\section{OPEN ACCESS}

Edited by:

Giovanna Gambarotta Università di Torino, Italy

Reviewed by: Pablo Hector Horacio Lopez, Instituto de Investigación Médica Mercedes y Martín Ferreyra (INIMEC),

Argentina

Barbara Hausott,

Innsbruck Medical University, Austria

${ }^{*}$ Correspondence:

Jiasong Guo

jiasongguo@aliyun.com

Received: 21 July 2018 Accepted: 05 November 2018

Published: 20 November 2018

Citation:

Tan D, Wen J, Li L, Wang X, Qian C, Pan M, Lai M, Deng J, Hu X, Zhang $H$ and Guo J (2018) Inhibition of RhoA-Subfamily GTPases Suppresses Schwann Cell Proliferation Through Regulating AKT Pathway Rather Than ROCK Pathway.

Front. Cell. Neurosci. 12:437. doi: 10.3389/fncel.2018.00437
Inhibiting RhoA-subfamily GTPases by C3 transferase is widely recognized as a prospective strategy to enhance axonal regeneration. When C3 transferase is administered for treating the injured peripheral nerves, Schwann cells (SCs, important glial cells in peripheral nerve) are inevitably impacted and therefore SC bioeffects on nerve regeneration might be influenced. However, the potential role of C3 transferase on SCs remains elusive. Assessed by cell counting, EdU and water-soluble tetrazolium salt-1 (WST-1) assays as well as western blotting with PCNA antibody, herein we first found that CT04 (a cell permeable C3 transferase) treatment could significantly suppress SC proliferation. Unexpectedly, using Y27632 to inhibit ROCK (the well-accepted downstream signal molecule of RhoA subfamily) did not impact SC proliferation. Further studies indicated that CTO4 could inactivate AKT pathway by altering the expression levels of phosphorylated AKT ( $p-A K T$ ), PI3K and PTEN, while activating AKT pathway by IGF-1 or SC79 could reverse the inhibitory effect of CTO4 on SC proliferation. Based on present data, we concluded that inhibition of RhoA-subfamily GTPases could suppress SC proliferation, and this effect is independent of conventional ROCK pathway but involves inactivation of AKT pathway.

Keywords: Schwann cell, proliferation, RhoA-subfamily GTPase, C3 transferase, AKT, ROCK

\section{INTRODUCTION}

Rho family small guanosine triphosphatases (Rho-GTPases) are essential in the regulation of diverse cellular functions such as regulation of actin cytoskeleton, vesicular trafficking and transcriptome dynamics (Hu and Selzer, 2017; Nomikou et al., 2017). Rho-GTPases cycle between an active GTP-bound and an inactive GDP-bound form, regulated by the opposing actions of guanine nucleotide exchange factors (GEFs) and GTPase-activating proteins (GAPs; Bai et al., 2015). The best characterized members of Rho family are classified into three subgroups, the RhoA (RhoA, B, C), Cdc42 (Cdc42, Tc10 and TcL) and Rac (Rac1, 2, 3 and RhoG) subfamilies, respectively (Erschbamer et al., 2005). RhoA subfamily is widely recognized as a crucial molecular switch to initiate growth cone collapse and inhibit axonal regrowth in the nervous system (Antoine-Bertrand et al., 2011; Matsukawa et al., 2018). 
Peripheral nerve injury (PNI) is a common global clinical problem which involves approximate $2.8 \%$ clinic trauma patients, and it significantly affects the life quality of patients and arouses an enormous socioeconomic burden (Wang et al., 2017). Recently, inhibiting RhoA subfamily has been accepted as a prospective strategy to facilitate axonal regrowth and functional recovery after PNI (Hiraga et al., 2006; Auer et al., 2012; Hynds, 2015; Joshi et al., 2015). Due to C3 transferase is able to selectively inactivate RhoA-subfamily GTPases, it is widely used to promote neural regeneration (Auer et al., 2012, 2013; Zhou et al., 2012; Forgione and Fehlings, 2014; Gutekunst et al., 2016).

Peripheral nerves are composed not only of axons but also of Schwann cells (SCs), which wrap around the axons and form myelin sheath (Tricaud, 2017). SCs are the first cells activated following PNI and play vital roles in nerve regeneration through dedifferentiation and proliferation (Pan et al., 2017). The proliferated SCs can organize the clearance of broken axons and myelin debris by promoting macrophage recruitment or via phagocytosis by themselves, secrete neurotrophins to facilitate the axonal regrowth, form bands of Bunger in the distal stump to provide a permissive microenvironment for axon regeneration and ensuing remyelination (Monk et al., 2015; Jessen and Mirsky, 2016; Wong et al., 2017). Thus, SC proliferation is regarded as a crucial part of the nerve injury and regeneration (Jessen et al., 2015). When C3 transferase is administered to promote the axonal regeneration in the injured peripheral nerves, SCs are inevitably impacted and their bioeffects on nerve regeneration might be influenced. However, the potential roles of C3 transferase on SCs remain elusive. To figure out this issue, the present project was firstly designed to reveal the effect of CT04 (a cell permeable C3 transferase) on SC proliferation and then the underlying mechanisms were also studied.

\section{MATERIALS AND METHODS}

\section{Primary Cultures of Schwann Cells}

The procedures of this study were performed in accordance with National Institutes of Health (NIH) guidelines for the care and use of laboratory animals (NIH Publications) and approved by the Animal Experimental Ethics Committee of the Southern Medical University (SMU), Guangdong Province, China. All efforts were made to minimize animal suffering and usage. Primary rat SCs were isolated and cultured according to our previous reports (Wen et al., 2017a,b). Briefly, spinal and sciatic nerves were aseptically isolated from 3day to 5-day postnatal Sprague-Dawley (SD) rats (provided by the Experimental Animal Center of SMU). The collected nerves were dissociated by $0.25 \%$ Trypsin-EDTA (Gibco) at $37^{\circ} \mathrm{C}$ for $30 \mathrm{~min}$ and single cells were obtained by gentle pipetting. Following digestion and dissociation, the cells were centrifuged for $10 \mathrm{~min}$ at $1,000 \mathrm{rpm}$ and re-suspended in DMEM/F12 (Corning) containing 10\% fetal bovine serum (FBS, Corning). Then cells were plated onto poly-L-lysine (PLL, Sigma-Aldrich)-coated petri dish (Jet Biofil). The next day, $10 \mu \mathrm{M}$ of cytosine arabinoside (Sigma-Aldrich) was added into the medium and incubated with the cells to eliminate fibroblasts. Forty-eight hours later, the medium was replaced by SC medium (DMEM/F12) containing 3\% FBS, $3 \mu \mathrm{M}$ forskolin (Sigma-Aldrich), $10 \mathrm{ng} / \mathrm{ml}$ heregulin (PeproTech) and $100 \mathrm{mg} / \mathrm{ml}$ penicillin-streptomycin (Gibco) to expand the cells. And all experiments of the present study were routinely performed using SCs collected at passages 3-5th. In designed experiments, $2 \mu \mathrm{g} / \mathrm{ml} \mathrm{CT04}$ (RhoA-subfamily GTPases inhibitor, Cytoskeleton), $50 \mu \mathrm{M}$ Y27632 (ROCK inhibitor, Selleck), $150 \mathrm{ng} / \mathrm{ml}$ IGF-1 (AKT activator, PeproTech) or $20 \mu \mathrm{M}$ SC79 (AKT activator, Selleck) was added into the culture medium and maintained for $24 \mathrm{~h}$.

\section{Immunofluorescence Staining}

To characterize the primary isolated cells, the cultured cells of passage 3 were fixed by $4 \%(\boldsymbol{w} / \boldsymbol{v})$ paraformaldehyde for $20 \mathrm{~min}$ and washed three times with $0.01 \mathrm{M}$ PBS. The fixed cells were permeabilized by $0.5 \%$ Triton X-100 (Sigma) for $30 \mathrm{~min}$ and then blocked with $5 \%$ bovine serum albumin (BSA, GBCBIO Technologies) in PBS for $1 \mathrm{~h}$ at room temperature, followed by the incubation with primary antibodies diluted in $1 \%$ BSA overnight at $4^{\circ} \mathrm{C}$. The dilutions of the primary antibodies are as follows: rabbit anti-GFAP (1:400, Sigma-Aldrich); mouse anti-S100 (1:200, Millipore); and mouse anti-P75 (1:400, Millipore). Alexa 488 fluorescent conjugated secondary antibodies (1:400, Molecular Probes) were applied for $2 \mathrm{~h}$ at room temperature, and the nuclei were counterstained by $1 \mu \mathrm{g} / \mathrm{ml} \mathrm{4}^{\prime}$,6-diamidino-2-phenylindole (DAPI, Sigma) for 2 min. After immunofluorescence staining, the cultures were mounted using the anti-fading mounting medium (Vector) and images were captured with a fluorescent microscope (Leica).

\section{Schwann Cell Proliferation Assays}

\section{EdU Incorporation Assay}

The EdU incorporation assay was conducted according to the manufacturer's instructions (RiboBio). In brief, the cells were seeded at $1 \times 10^{4} /$ well in 96-well plates and incubated overnight to allow cell adherence. Cells were exposed to various drug treatments as designed for $24 \mathrm{~h}$ and then incubated with $50 \mu \mathrm{M}$ EdU labeling reagent for $3 \mathrm{~h}$ prior to fixation. Following permeabilization in $0.5 \%$ Triton $\mathrm{X}-100$, the cells underwent EdU staining. The cell nuclei were counterstained with DAPI. EdU-positive nuclei were determined under a fluorescence microscope (Leica). Five images were captured at the center and four quadrants in each plate using a fluorescent microscope. The EdU positive ratio was calculated as the number of EdU-positive cells divided by the number of total cells (positive for DAPI). Meanwhile, the cell density of each group was calculated and defined as the number of cells (positive for DAPI) in each captured image. The number of cells was counted using Image-Pro Plus software (Media Cybernetics).

\section{WST-1 Assay}

The cell proliferation was also evaluated by the water-soluble tetrazolium salt-1 (WST-1) assay using a Quick Cell Proliferation Assay Kit II (Abcam). SCs were prepared as described above (EdU incorporation assay). According to the manufacturer's instructions, $10 \mu \mathrm{l}$ of the WST reagent was added to each 
well of the 96 -well plates for $3 \mathrm{~h}$ at $37^{\circ} \mathrm{C}$. The absorbance was measured at $450 \mathrm{~nm}$ by using a microplate reader (BioTek). A background $_{\text {is the absorbance of culture medium plus }}$ WST in the absence of cells. The actual absorbance of the control cells $\left(\mathrm{A}_{\text {control }}\right)$ is the absorbance of $\mathrm{A}_{\text {control }}$ minus the absorbance of $A_{\text {background. The actual absorbance of the treated }}$ cells $\left(A_{\text {experimental }}\right)$ is the absorbance of $A_{\text {experimental minus the }}$ absorbance of $A_{\text {background }}$.

\section{Western Blotting}

For western blotting, SCs were treated with various drugs as designed for $24 \mathrm{~h}$. The subjected cells were washed twice with ice-cold PBS, scraped and lysed in RIPA buffer (GBCBIO) containing protease inhibitor cocktail (1:100, Cell Signaling). Lysates were incubated on ice for $30 \mathrm{~min}$ and centrifuged $\left(14,000 \mathrm{rpm}, 20 \mathrm{~min}, 4^{\circ} \mathrm{C}\right)$ in order to collect the supernatant. Extracts were combined with SDS-PAGE sample buffer (400 mM Tris/ $\mathrm{HCl}, \mathrm{pH} 6.8,10 \%$ SDS, $50 \%$ glycerol, $500 \mathrm{mM}$ DTT, $2 \mu \mathrm{g} / \mathrm{ml}$ bromophenol blue) and denatured by boiling for $10 \mathrm{~min}$. Equal protein samples were loaded and resolved by $10 \%$ SDS-PAGE gels and transferred to polyvinylidene difluoride (PVDF) membrane (Bio-Rad) by liquid transfer. Membranes were blocked with 5\% BSA for $1 \mathrm{~h}$ at room temperature and incubated with primary antibodies overnight at $4^{\circ} \mathrm{C}$. The following primary antibodies were used in western blotting: rabbit anti-GAPDH (1:3,000, Multi Science); rabbit anti-PCNA (1:500, Cell Signaling Technology); rabbit anti-PTEN (1:1,000, Cell Signaling Technology); rabbit anti-PI3K (1:1,000, Cell Signaling Technology); rabbit antiphospho-AKT (1:1,000, Cell Signaling Technology); rabbit anti-AKT (1:1,000, Cell Signaling Technology). After washed three times with Tris-buffered saline containing $0.05 \%$ Tween-20, the membranes were incubated with respective
HRP-conjugated secondary antibodies (1:2,000, Cell Signaling Technology) for $2 \mathrm{~h}$ at room temperature. Immunoreactive proteins bands were detected and imaged by enhanced chemiluminescence (ECL, Millipore) using Lumazone system (Roper). After exposure, membranes were washed by stripping buffer (Thermo) for further incubation of another antibody if needed. Integrated optical density (IOD) of each lane was quantified with Image-Pro Plus software and the expression levels of targeted proteins were normalized by the IOD of GAPDH.

\section{Cytotoxicity Assay by Live/Dead Cell Staining}

In order to assess whether the effect of CT04 on cell proliferation is attributed to the cytotoxicity of CT04, an assay with Live/Dead cell staining kit (BestBio) was conducted according to the manufacturer's instructions. In brief, the cells were seeded at $1 \times 10^{4}$ /well in 96-well plates and incubated overnight to allow cell adherence. The culture media were supplemented with $2 \mu \mathrm{g} / \mathrm{ml} \mathrm{CT04}$ or $30 \%$ DMSO for $24 \mathrm{~h}$ and then incubated for $30 \mathrm{~min}$ with the Live/Dead cell staining solution which was prepared with the reagents $\mathrm{A}, \mathrm{B}$ and $\mathrm{C}$ of the kit. After that, the cultures were observed with a fluorescence microscope to detect the live cells (stained by Calcein-AM with green fluorescence) and dead cells (stained by PI with red fluorescence). Five images were captured at the center and four quadrants in each culture. Then the live cell ratio was calculated as the number of Calcein-AM positive cells divided by the number of total cells (Calcein-AM-positive cells plus PI-positive cells).

\section{Statistical Analysis}

All statistical analyses and calculations were carried out using SPSS 20.0 software (IBM, Armonk, NY, USA). Statistics for
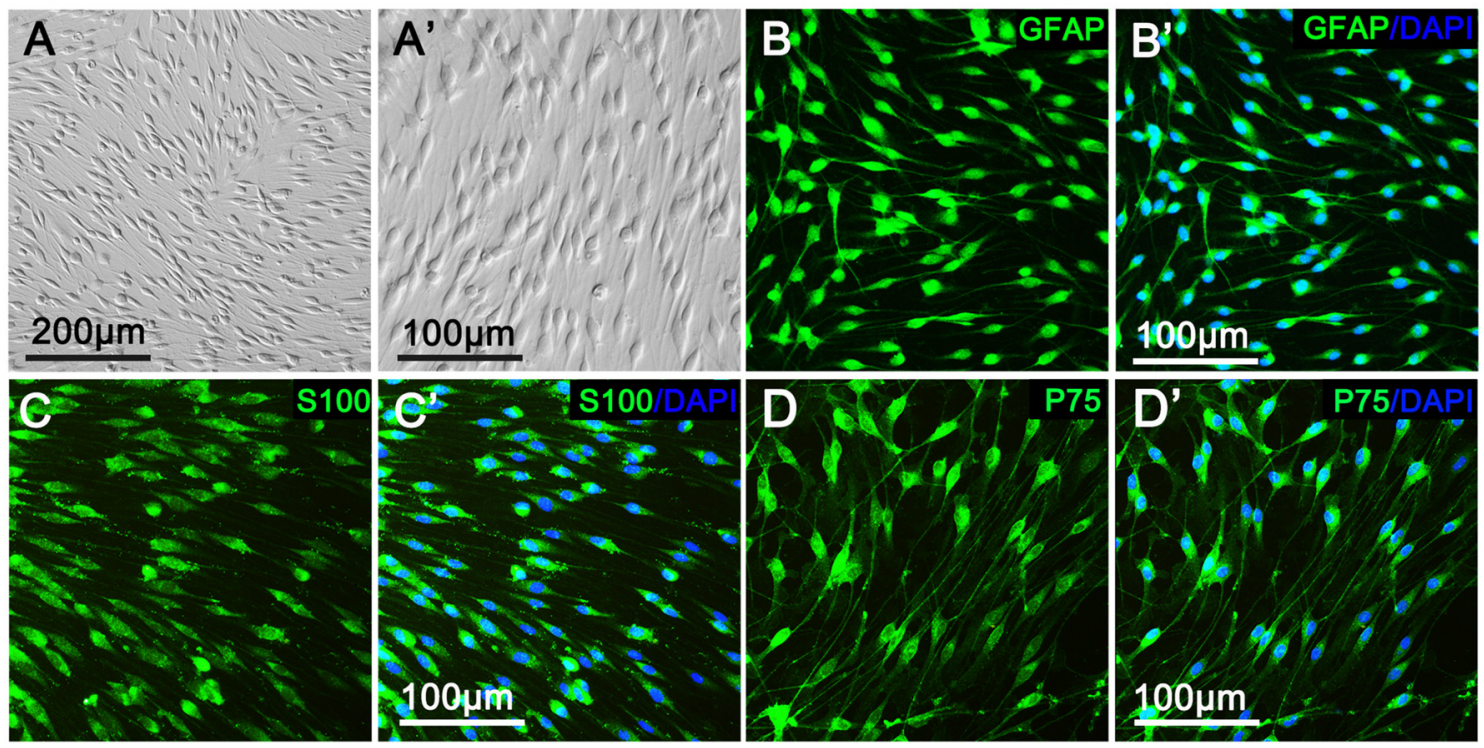

FIGURE 1 | Characterization and identification of primary cultured Schwann cells (SCs). (A,A') Primary cultured SCs showed a bipolar spindle shape under phase contrast microscope. More than 95\% of the cells were positive for the SC specific markers, including GFAP (B,B'), S100 (C,C') and P75 (D,D') 
multiple comparisons were generated using one-way ANOVA followed by Bonferroni post hoc tests. Independent samples $t$-test was used to analyze values between two groups. All statistical graphs were plotted with the Graph Pad Prism 6.0 (Graph Pad software), and quantitative data were presented as mean \pm standard deviation (SD). Differences were considered statistically significant with $P$-value $<0.05$.

\section{RESULTS}

\section{CT04 Suppresses Schwann Cell Proliferation}

As shown in Figure 1, primary cultured SCs exhibited a bipolar spindle shape (Figures $\mathbf{1 A}, \mathbf{A}^{\prime}$ ) and were positive for SC-specific markers, including GFAP, S100 and P75 (Figures 1B-D, $\mathbf{B}^{\prime}-\mathbf{D}^{\prime}$ ). To illustrate the effect of CT04 on SC proliferation, SCs were treated by CT04 for $24 \mathrm{~h}$. Then, the EdU and WST-1 measurements were conducted. The EdU assay displayed that the ratio of EdU positive cells was significantly reduced in the presence of CT04 (Figures 2A-G). Furthermore, the statistical analysis of cell density indicated that the number of total cells was also decreased by CT04 treatment (Figure $2 \mathbf{H}$ ). The WST-1 results revealed that CT04 markedly decreased the absorbance value compared to the control group (Figure 2I). Meanwhile, the level of PCNA, determined using western blotting, was remarkably down-regulated by CT04 treatment (Figures 2J,K). Taken together, all these results confirm that CT04 can suppress SC proliferation.

Further experiment with Live/Dead cell staining assay was designed to test the potential cytotoxicity of CT04 in the SC cultures. In order to confirm the efficiency and reliability of this assay, we set up a positive control (30\% DMSO treatment) and a negative control (no drug treatment) to do the parallel experiment with the CT04 treatment. The results illustrated that the overwhelming majority of cells in both of the negative control group and CT04 group were live cells, only very few of cells in these two groups were dead cells (Figures 3A-F,J). However, almost all cells in the positive control group were dead cells (Figures 3G-J). These results demonstrate that the effect of CT04 on SC proliferation is not caused by cytotoxicity.

\section{Inhibition of ROCK Does Not Affect Schwann Cell Proliferation}

To identify whether CT04 modulates SC proliferation through ROCK which is the most well-known downstream effector of RhoA-subfamily GTPases, the SCs were treated with Y27632 (a widely used specific ROCK inhibitor) for $24 \mathrm{~h}$. Unexpectedly,
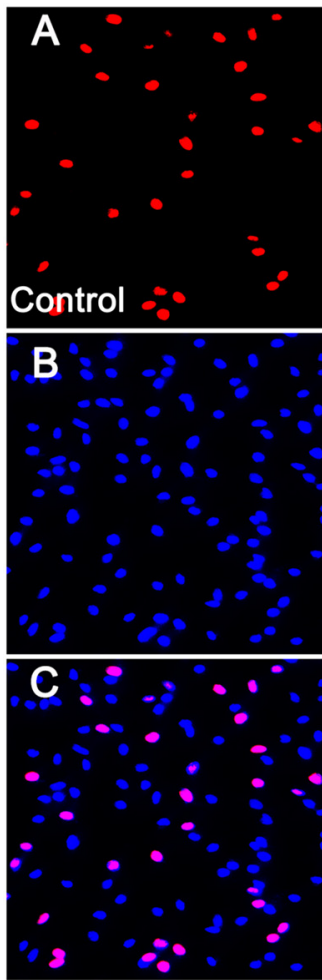
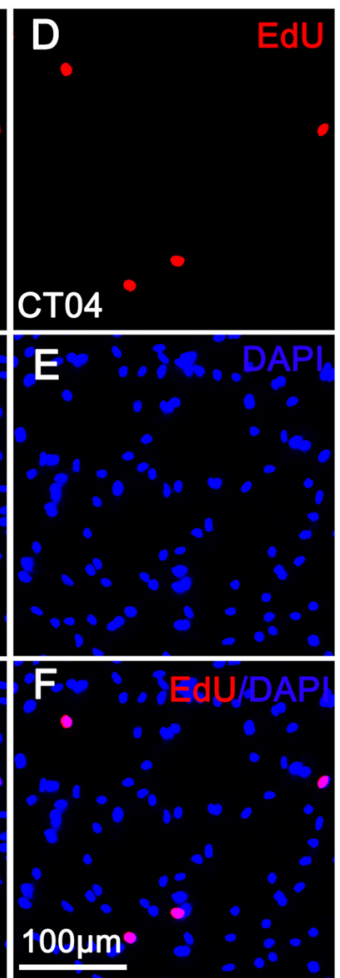

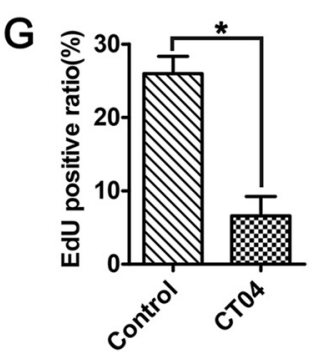

$\mathrm{H}$

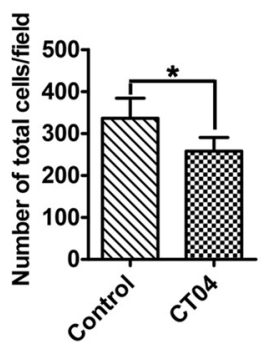

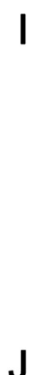
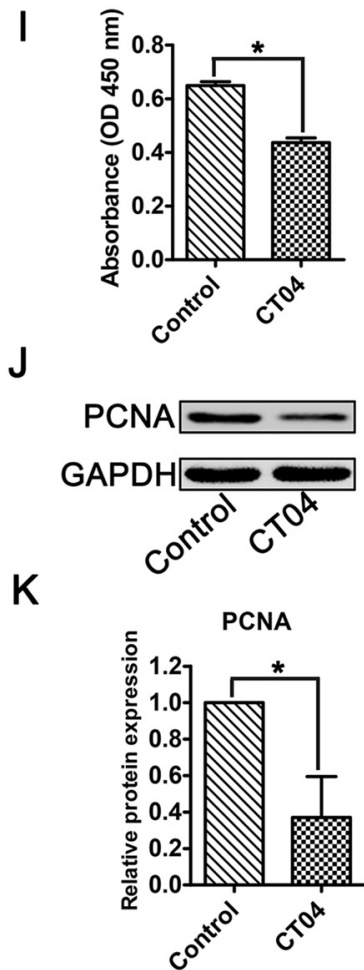

FIGURE 2 | CT04 suppresses SC proliferation. (A-G) EdU staining showed that EdU positive ratio was suppressed by CT04 treatment $\left(n=15,{ }^{*} P<0.05\right)$. (H) Statistical graph of cell density indicated that the number of total cells (positive for $4^{\prime}$,6-diamidino-2-phenylindole (DAPI)) was decreased in the presence of CT04 ( $n=15,{ }^{*} P<0.05$ ). (I) Water-soluble tetrazolium salt-1 (WST-1) measurement revealed that CT04 markedly decreased the absorbance value compared to the control group $\left(n=4,{ }^{*} P<0.05\right)$. ( $\left.\mathbf{J}, \mathbf{K}\right)$ Western blotting displayed that the expression of PCNA was remarkably down-regulated in the CT04 treated cells $(n=6$, $\left.{ }^{*} P<0.05\right)$. The blots were cropped from different parts of the same gel. The expression level of PCNA in the control group was normalized to 1. 


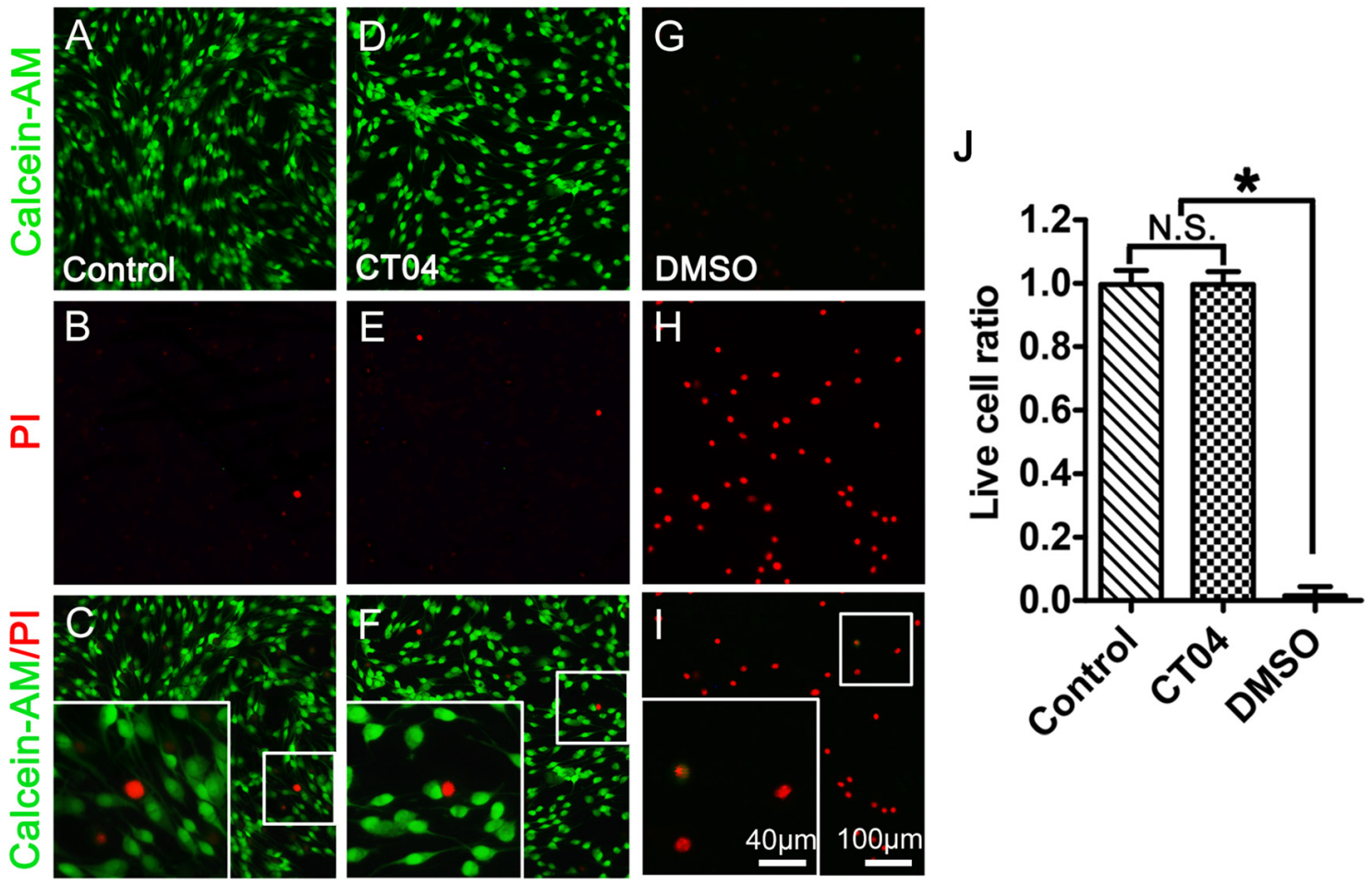

FIGURE 3 | CT04 does not induce toxic effect on SC. The cytotoxicity of CT04 was evaluated using Live/Dead cell staining. (A-J) The Live/Dead cell staining and statistical diagrams suggested that addition of CTO4 did not induce cell death in the SCs cultures $(n=20$, $* P<0.05)$. N.S. as non-significance.

Y27632 did not result in the same effect on SC proliferation as CT04. As shown in Figure 4, the EdU assay indicated that the ratio of EdU positive cells as well as the cell density was not affected in the presence of Y27632 (Figures 4A-G,H). Meanwhile, WST-1 assay showed no difference in absorbance value between Y27632 and control groups (Figure 4I). In addition, the expression level of PCNA was similar between two groups (Figures 4J,K). These data strongly implicate that ROCK is not involved in the regulation of SC proliferation.

\section{CT04 Inactivates AKT Signaling Pathway}

According to previous reports (He et al., 2011; Chen et al., 2016; Wu et al., 2016), AKT pathway is one of the most important pathways involved in regulating SC proliferation. To determine whether this pathway is responsible for mediating the CT04-induced suppression on SC proliferation, the total AKT, phosphorylated AKT (p-AKT) as well as AKT's critical upstream regulator-PI3K (Gaesser and Fyffe-Maricich, 2016) and its crucial inhibitor-PTEN (Liu et al., 2017) were detected. Western blotting verified that the $\mathrm{p}$-AKT was significantly decreased in CT04 group, while the total AKT was unaffected (Figures 5A,B). Furthermore, the expression of PI3K was down-regulated in the presence of CT04 (Figures 5C,D), while the expression of PTEN was up-regulated (Figures 5E,F). These data drew us to hypothesize that AKT pathway might be involved in the inhibitory effect of CT04 on SC proliferation.

\section{Activation of AKT Reverses the Inhibitory Effect of CT04 on SC Proliferation}

To ascertain the potential role of AKT pathway on CT04-mediated inhibition of SC proliferation, we treated the SCs with CT04 in the presence or absence of AKT activators (IGF-1 (Jo et al., 2012; Sabater et al., 2017) or SC79 (Wu et al., 2017; Yang et al., 2018; Zhou et al., 2018)), respectively. Western blotting indicated that IGF-1 dramatically increased the level of p-AKT while the level of total AKT was not affected (Figures 6A,B). Subsequently, the EdU assay showed that CT04-induced suppression of EdU positive ratio of SCs and the cell density were obviously restored by IGF-1 (Figures 6C-L,M). The effect of IGF-1 on SC proliferation was also verified by the change in absorbance value detected by WST-1 measurement (Figure 6N). What's more, the down-regulation of PCNA expression caused by CT04 treatment was also significantly alleviated by IGF-1 as shown in western blots (Figures 60,P). Similar findings were observed when SCs were treated with CT04 in the presence of SC79, another specific activator of AKT. The results of cell density, EdU, WST-1 and western blotting assays also revealed that the addition of SC79 partly reversed CT04-mediated suppression of SC proliferation (Figure 7).

\section{DISCUSSION}

Collectively, overall data of the present study demonstrate that: (1) data got from the assessments of cell density, EdU, 

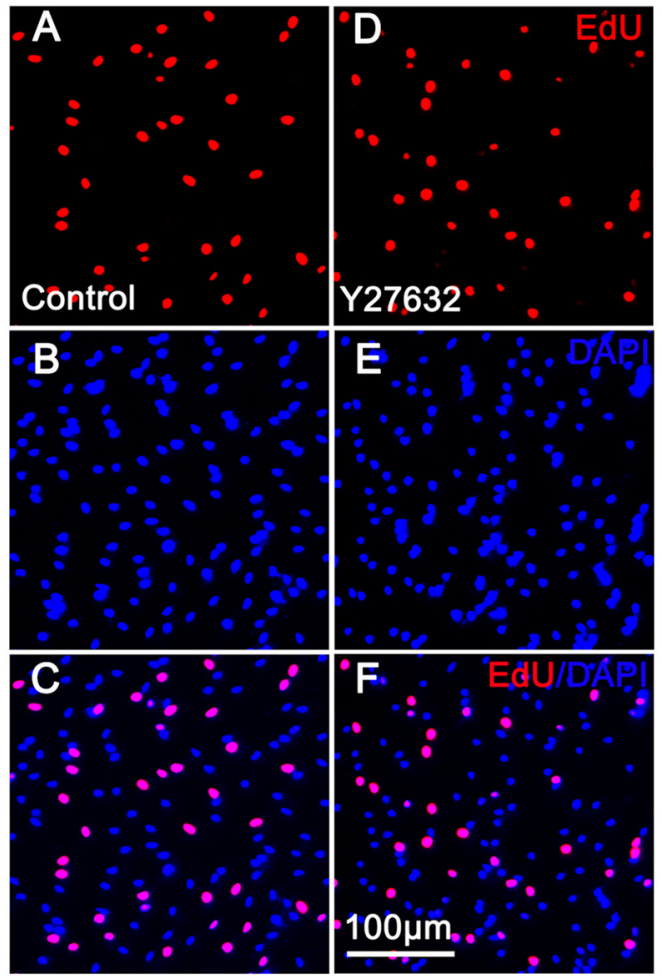
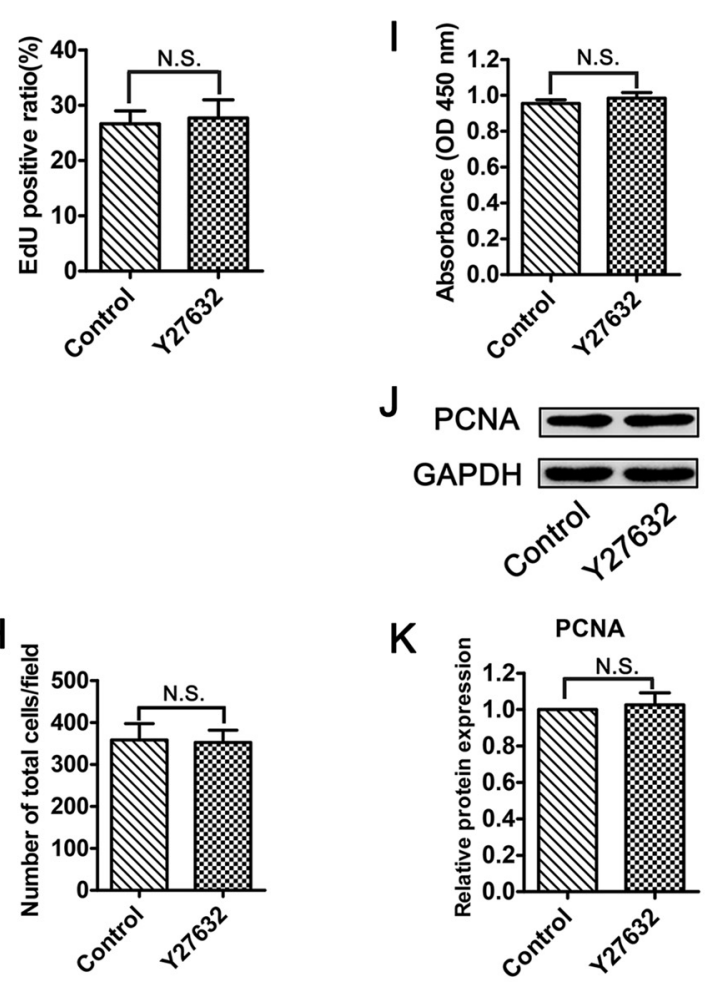

FIGURE 4 | Inhibition of ROCK does not affect SC proliferation. (A-G) EdU assay showed that EdU positive ratio was not affected by Y27632 treatment ( $n=15)$. (H) Statistical diagram of cell density suggested that the number of total cells was not altered in the presence of Y27632 ( $n=15$ ). (I) WST-1 measurement revealed that there was no significant difference in the absorbance value between the control group and Y27632 group $(n=4)$. (J,K) Western blotting indicated that the expression of PCNA was unaffected in the Y27632 treated cells $(n=6)$. The blots were cropped from different parts of the same gel. The expression level of PCNA in the control group was normalized to 1. N.S. as non-significance.

WST-1 and PCNA expression indicate that the inhibition of RhoA-subfamily GTPases by C3 transferase (CT04) can suppress the SC proliferation; (2) Live/Dead cell staining assay indicates CT04 does not induce cell death in the SCs cultures, which means the effect of CT04 on SC proliferation was not related to the cytotoxicity; (3) Y27632 (a widely used specific ROCK inhibitor) does not affect the SC proliferation. By which excludes the possibility of RhoA-subfamily GTPases regulating SC proliferation via ROCK pathway; (4) the level of p-AKT is significantly decreased in the CT04 treated SCs, while the total AKT is unaffected. Since AKT activation (phosphorylation) is involved in regulating the proliferation of many kinds of cells including SCs, these results suggest AKT inactivation might play a role in CT04 negative effects on SC proliferation; (5) CT04 treatment results in down-regulation of PI3K and up-regulation of PTEN, which can confirm and verify the results of CT04 suppressing the AKT activation; and (6) reversing the AKT activation by IGF-1 or SC79 (two kinds of widely used AKT activators) can significantly alleviate the inhibitory effect of CT04 on SC proliferation. These data confirm that AKT pathway is involved in the mechanisms of CT04-induced suppression on SC proliferation.

Since SCs are important glial cells in peripheral nerve and they have attractive application prospects in cell transplantation for the therapy of nervous system injury, the biology and application of SCs have been attracting a great deal of attention (Belin et al., 2017). Recent decades, lots of publications focused on the study of SC proliferation (Atanasoski et al., 2004; Deng et al., 2017; Pan et al., 2017; Piñero et al., 2017). As molecular switches that control the organization and dynamics of the actin cytoskeleton, Rho GTPases are considered to be key regulators of proliferation of various cells ( $\mathrm{Hu}$ et al., 2014; Wang et al., 2014). Our previous study indicated that lentivirus-mediated RhoA knocking down to reduce the total protein level of RhoA could significantly slack the proliferation of SCs (Wen et al., 2017a). In this study, we aimed to reveal whether inhibiting the activation of RhoA-subfamily GTPases can impact the SC proliferation. CT04 is able to specifically and irreversibly inhibit activation of RhoA-subfamily GTPases by ADP ribosylation, our recent study proved CT04 treatment could reduce the level of activated GTP-RhoA in cultured SCs (Wen et al., 2018). Combined with the present data of cell density, EdU assay, WST-1 assessment and western blotting of PCNA, we can safely draw the conclusion that inhibition of RhoA-subfamily GTPases by CT04 can significantly slow down the SC proliferation.

Up to now, the most well-known downstream effector of RhoA-subfamily GTPases is ROCK. In existing reports, RhoA subfamily plays roles in various biologic effects including 
A

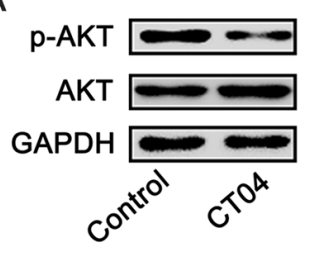

B

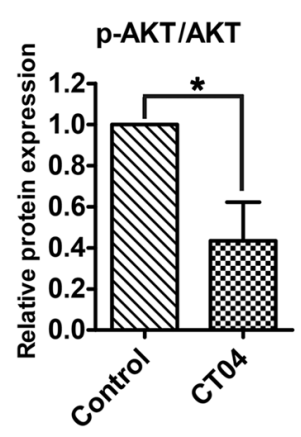

C

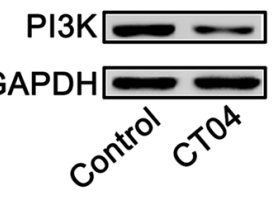

D

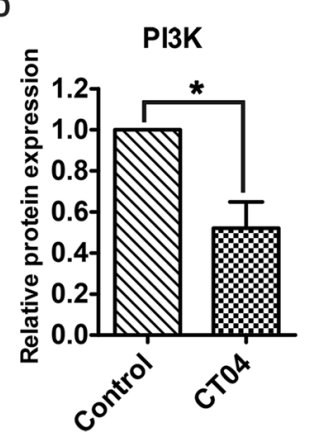

\section{E}

PTEN $-\longrightarrow$

GAPDH

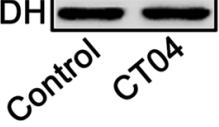

F

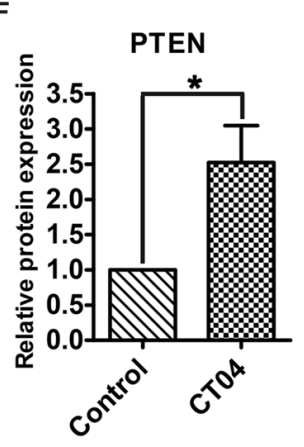

FIGURE 5 | CT04 regulates the AKT signaling pathway. (A,B) The western blots indicated that the phosphorylation of AKT was markedly decreased in the presence of CT04 ( $n=12, * P<0.05)$. (C-F) Immunoblot assays and statistical diagrams also revealed that addition of CT04 resulted in down-regulation of PI3K and up-regulation of PTEN $\left(n=4,{ }^{*} P<0.05\right)$. The blots were cropped from different parts of the same gel. The expression levels of target proteins in the control group were normalized to 1.

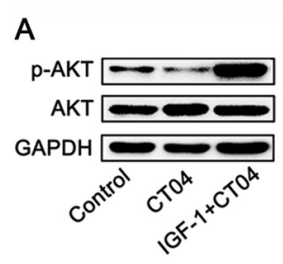

B

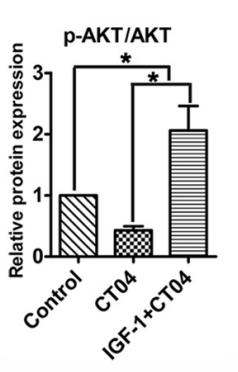

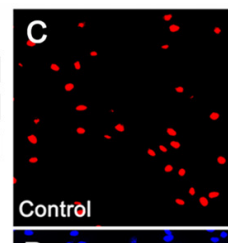

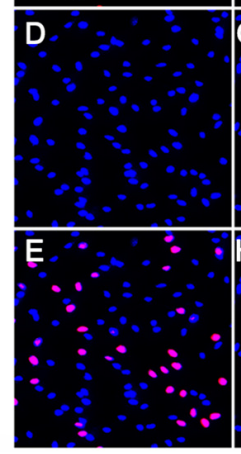

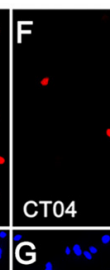

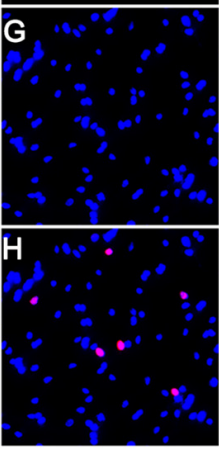

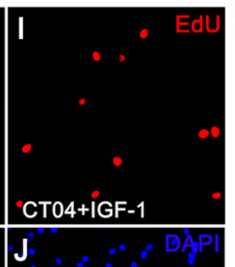

$\mathrm{J}$

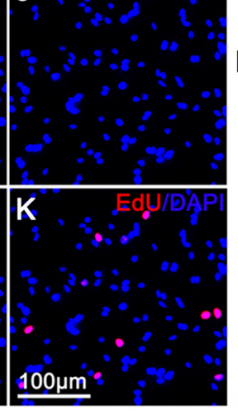

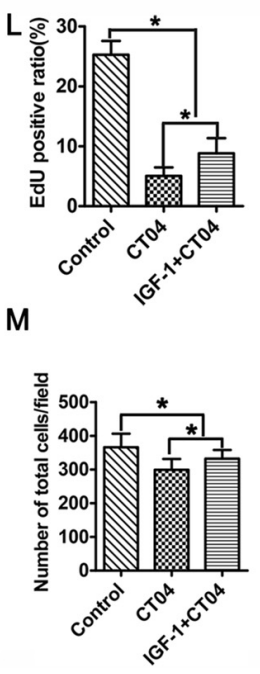

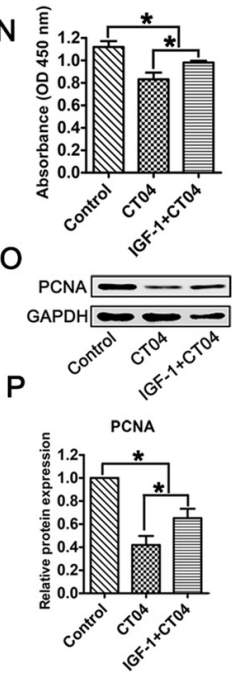

FIGURE 6 | Activation of AKT by IGF-1 reverses the inhibitory effect of CTO4 on SC proliferation. The SCs were treated with CT04 in the presence or absence of IGF-1 for 24 h. (A,B) Western blotting was performed to confirm the effect of IGF-1 on activation of AKT. GAPDH was used as the loading control $\left(n=4,{ }^{*} P<0.05\right)$. (C-L) Analysis of EdU incorporation indicated that IGF-1 partly restored the declined EdU positive ratio in CT04 treated cells $\left(n=15,{ }^{*} P<0.05\right)$. (M) Statistics of cell density displayed that CT04-mediated inhibition of SC density was reversed by IGF-1 $\left(n=15,{ }^{*} P<0.05\right)$. (N) WST-1 assay revealed that IGF-1 treatment partly restored the change in absorbance value caused by CTO4 ( $\left.n=4,{ }^{*} P<0.05\right)$. (O) Western blots showed that application of IGF-1 up-regulated the expression of PCNA in the CT04 treated cells. The blots were cropped from different parts of the same gel. (P) Statistical diagram of relative protein expression of PCNA ( $n=4$, $\left.{ }^{*} P<0.05\right)$. The expression levels of target proteins in the control group were normalized to 1.

cell proliferation always by regulating actin dynamics via ROCK pathway. Thus, we naturally consider CT04 may also influence SC proliferation through ROCK pathway. However, the treatment of Y27632, a widely used inhibitor of ROCK, has no significant impact on the parameters of SC proliferation including cell density, EdU, WST-1 and western blotting of PCNA. Therefore, ROCK is impossible taking part in the regulation of RhoA subfamily on SC proliferation. 

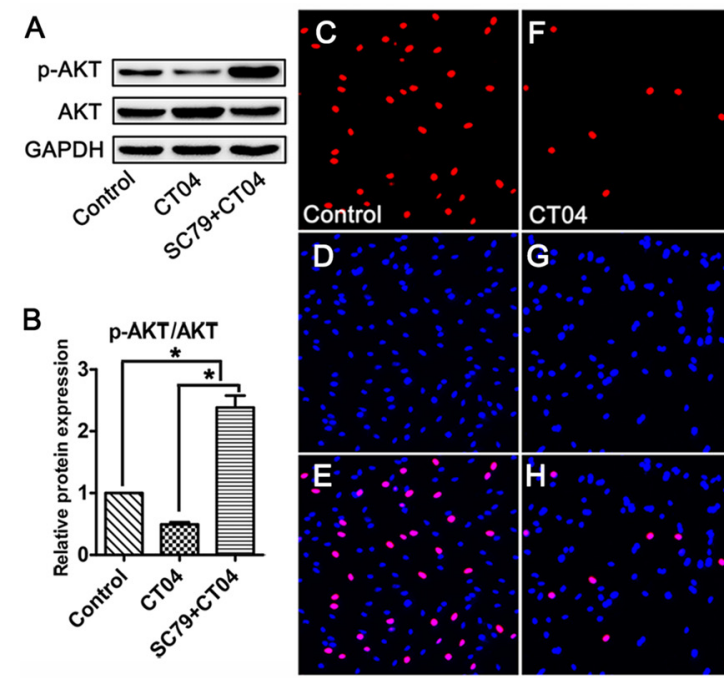
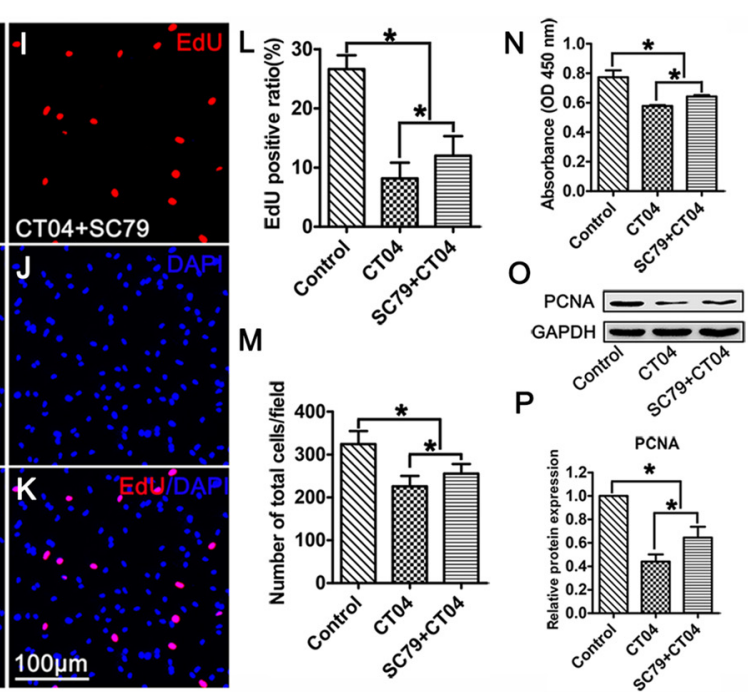

FIGURE 7 | Activation of AKT by SC79 counteracts the inhibitory effect of CTO4 on SC proliferation. SCs were treated with DMSO as vehicle control. The SCs were treated with CT04 with or without SC79 for 24 h. (A,B) Western blotting was performed to confirm the effect of SC79 on activation of AKT ( $n=4$, $\left.{ }^{*} P<0.05\right)$. (C-L) The EdU incorporation assay suggested that application of SC79 increased the EdU positive ratio in CT04 treated cells ( $n=15$, $\left.{ }^{*} P<0.05\right)$. (M) The statistics showed that CT04-mediated suppression of SC density was partly restored by SC79 $(n=15, * P<0.05)$. (N) Assessment of cell proliferation by WST-1 assay displayed that SC79 counteracted the inhibitory effect of CT04 on SC proliferation $\left(n=4,{ }^{*} P<0.05\right)$. (O,P) Western blots and statistical data indicated that the addition of SC79 increased the expression of PCNA in the CT04 treated cells $(n=4, * P<0.05)$. The blots were cropped from different parts of the same gel. The expression levels of target proteins in the control group were normalized to 1.

AKT pathway is demonstrated as an important signaling pathway in the regulation of cell proliferation, including SCs (Wu et al., 2016; Liu et al., 2017; Zhao et al., 2017). This drew us to think whether CT04 treatment modulated SC proliferation via AKT pathway. In order to test this hypothesis, the phosphorylation of AKT was firstly evaluated. The results demonstrated that CT04 treatment significantly reduced the level of $\mathrm{p}$-AKT. Moreover, the expression level of PI3K, which is the crucial positive upstream molecule of AKT, was markedly decreased. It has been shown in several researches that in the PTEN/PI3K/AKT signaling pathway, PI3K can catalyze 3,4,5phosphatidylinositol trisphosphate phosphorylation and then activate AKT to promote the proliferation of cells (Zhang et al., 2015). In addition, we found that the expression of PTEN was dramatically up-regulated. PTEN is an important negative regulator of AKT pathway, it can antagonize PI3K and then weaken the activation of AKT (Zhang et al., 2015; Ahmed et al., 2016). Therefore, the up-regulation of PTEN and down-regulation of PI3K further confirmed that AKT pathway could be inactivated by CT04. To further validate whether the inactivation of AKT pathway was responsible for CT04-mediated suppression of SC proliferation, two kinds of activators of AKT pathway (IGF-1 and SC79) were used to do the rescue experiments. As expected, both IGF-1 and SC79 could reverse the AKT inactivation by CT04 and significantly alleviate the inhibitory effect of CT04 on SC proliferation.

Taken together, we can conclude that: (1) C3 transferase (CT04) treatment can significantly suppress the SC proliferation. Considering SCs are important glial cells in peripheral nerves and C 3 transferase is widely used to promote axonal regeneration in the injured peripheral nerve, the present study indicates further studies are needed to explore new strategies to avoid this side-effect when using this drug to treat the injured nerve; and (2) the effect of CT04 on SC proliferation involves the AKT pathway. While ROCK, the most well-known downstream molecule of Rho subfamily, is independent of the effect of CT04 on the SC proliferation. Considering the complicate signaling networks of Rho GTPases in most kinds of cells, and combining with present data, we believe that AKT is not the only downstream of RhoA subfamily on SC proliferation. The clear mechanism needs further studies in the future.

\section{AUTHOR CONTRIBUTIONS}

JG and DT designed the study. DT, JW, LL and XW performed experiments. CQ, MP and ML participated in collecting and analyzing experimental data. JD, XH and $\mathrm{HZ}$ contributed for double blind analyzing data and doing statistics. The manuscript was written by JG, DT and reviewed by all authors.

\section{FUNDING}

This work is supported by the National Natural Science Foundation of China (81870982, 81571182 and 81371354), the Program for Changjiang Scholars and Innovative Research Team in University (IRT-16R37), National Key Basic Research Program of China (2014CB542202), the Science and Technology Project of Guangdong Province (2015A020212024) and Natural Science Foundation of Guangdong Province (2017A030312009). 


\section{REFERENCES}

Ahmed, M. W., Kayani, M. A., Shabbir, G., Ali, S. M., Shinwari, W.-U.-D., and Mahjabeen, I. (2016). Expression of PTEN and its correlation with proliferation marker Ki-67 in head and neck cancer. Int. J. Biol. Markers 31, e193-e203. doi: 10.5301/jbm.5000196

Antoine-Bertrand, J., Villemure, J.-F., and Lamarche-Vane, N. (2011). Implication of Rho GTPases in neurodegenerative diseases. Curr. Drug Targets 12, 1202-1215. doi: 10.2174/138945011795906543

Atanasoski, S., Notterpek, L., Lee, H.-Y., Castagner, F., Young, P., Ehrengruber, M. U., et al. (2004). The protooncogene Ski controls Schwann cell proliferation and myelination. Neuron 43, 499-511. doi: 10.1016/j.neuron. 2004.08.001

Auer, M., Allodi, I., Barham, M., Udina, E., Neiss, W. F., Navarro, X., et al. (2013). C3 exoenzyme lacks effects on peripheral axon regeneration in vivo. J. Peripher. Nerv. Syst. 18, 30-36. doi: 10.1111/jns5.12004

Auer, M., Schweigreiter, R., Hausott, B., Thongrong, S., Höltje, M., Just, I., et al. (2012). Rho-independent stimulation of axon outgrowth and activation of the ERK and Akt signaling pathways by $\mathrm{C} 3$ transferase in sensory neurons. Front. Cell. Neurosci. 6:43. doi: 10.3389/fncel.2012.00043

Bai, Y., Xiang, X., Liang, C., and Shi, L. (2015). Regulating Rac in the nervous system: molecular function and disease implication of Rac GEFs and GAPs. Biomed. Res. Int. 2015:632450. doi: 10.1155/2015/632450

Belin, S., Zuloaga, K. L., and Poitelon, Y. (2017). Influence of mechanical stimuli on Schwann cell biology. Front. Cell. Neurosci. 11:347. doi: 10.3389/fncel.2017. 00347

Chen, M.-S., Kim, H., Jagot-Lacoussiere, L., and Maurel, P. (2016). Cadm3 (Necl-1) interferes with the activation of the PI3 kinase/Akt signaling cascade and inhibits Schwann cell myelination in vitro. Glia 64, 2247-2262. doi: 10.1002/glia.23072

Deng, Y., Wu, L. M. N., Bai, S., Zhao, C., Wang, H., Wang, J., et al. (2017). A reciprocal regulatory loop between TAZ/YAP and G-protein Gas regulates Schwann cell proliferation and myelination. Nat. Commun. 8:15161. doi: 10.1038/ncomms15161

Erschbamer, M. K., Hofstetter, C. P., and Olson, L. (2005). RhoA, RhoB, RhoC, Rac1, Cdc42 and Tc10 mRNA levels in spinal cord, sensory ganglia and corticospinal tract neurons and long-lasting specific changes following spinal cord injury. J. Comp. Neurol. 484, 224-233. doi: 10.1002/cne.20471

Forgione, N., and Fehlings, M. G. (2014). Rho-ROCK inhibition in the treatment of spinal cord injury. World Neurosurg. 82, e535-e539. doi: 10.1016/j.wneu. 2013.01.009

Gaesser, J. M., and Fyffe-Maricich, S. L. (2016). Intracellular signaling pathway regulation of myelination and remyelination in the CNS. Exp. Neurol. 283, 501-511. doi: 10.1016/j.expneurol.2016.03.008

Gutekunst, C.-A., Tung, J. K., Mcdougal, M. E., and Gross, R. E. (2016). C3 transferase gene therapy for continuous conditional RhoA inhibition. Neuroscience 339, 308-318. doi: 10.1016/j.neuroscience. 2016.10.022

He, B., Liu, S.-Q., Chen, Q., Li, H.-H., Ding, W.-J., and Deng, M. (2011). Carboxymethylated chitosan stimulates proliferation of Schwann cells in vitro via the activation of the ERK and Akt signaling pathways. Eur. J. Pharmacol. 667, 195-201. doi: 10.1016/j.ejphar.2011.06.001

Hiraga, A., Kuwabara, S., Doya, H., Kanai, K., Fujitani, M., Taniguchi, J., et al. (2006). Rho-kinase inhibition enhances axonal regeneration after peripheral nerve injury. J. Peripher. Nerv. Syst. 11, 217-224. doi: 10.1111/j.1529-8027.2006. 00091.x

Hu, J., and Selzer, M. E. (2017). RhoA as a target to promote neuronal survival and axon regeneration. Neural Regen. Res. 12, 525-528. doi: 10.4103/1673-5374. 205080

Hu, X., Yu, J., Zhou, X., Li, Z., Xia, Y., Luo, Z., et al. (2014). A small GTPaselike protein fragment of Mycoplasma promotes tumor cell migration and proliferation in vitro via interaction with Rac1 and Stat3. Mol. Med. Rep. 9, 173-179. doi: 10.3892/mmr.2013.1766

Hynds, D. (2015). Subcellular localization of Rho GTPases: implications for axon regeneration. Neural Regen. Res. 10, 1032-1033. doi: 10.4103/1673-5374. 160064

Jessen, K. R., and Mirsky, R. (2016). The repair Schwann cell and its function in regenerating nerves. J. Physiol. 594, 3521-3531. doi: 10.1113/jp270874
Jessen, K. R., Mirsky, R., and Lloyd, A. C. (2015). Schwann cells: development and role in nerve repair. Cold Spring Harb. Perspect. Biol. 7:a020487. doi: 10.1101/cshperspect.a020487

Jo, H., Mondal, S., Tan, D., Nagata, E., Takizawa, S., Sharma, A. K., et al. (2012). Small molecule-induced cytosolic activation of protein kinase Akt rescues ischemia-elicited neuronal death. Proc. Natl. Acad. Sci. U S A 109, 10581-10586. doi: 10.1073/pnas.1202810109

Joshi, A. R., Bobylev, I., Zhang, G., Sheikh, K. A., and Lehmann, H. C. (2015) Inhibition of Rho-kinase differentially affects axon regeneration of peripheral motor and sensory nerves. Exp. Neurol. 263, 28-38. doi: 10.1016/j.expneurol. 2014.09.012

Liu, G.-L., Yang, H.-J., Liu, B., and Liu, T. (2017). Effects of microRNA-19b on the proliferation, apoptosis and migration of Wilms' tumor cells via the PTEN/PI3K/AKT signaling pathway. J. Cell. Biochem. 118, 3424-3434. doi: $10.1002 /$ jcb. 25999

Matsukawa, T., Morita, K., Omizu, S., Kato, S., and Koriyama, Y. (2018). Mechanisms of RhoA inactivation and CDC42 and Rac1 activation during zebrafish optic nerve regeneration. Neurochem. Int. 112, 71-80. doi: 10.1016/j. neuint.2017.11.004

Monk, K. R., Feltri, M. L., and Taveggia, C. (2015). New insights on Schwann cell development. Glia 63, 1376-1393. doi: 10.1002/glia.22852

Nomikou, E., Stournaras, C., and Kardassis, D. (2017). Functional analysis of the promoters of the small GTPases RhoA and RhoB in embryonic stem cells. Biochem. Biophys. Res. Commun. 491, 754-759. doi: 10.1016/j.bbrc.2017. 07.114

Pan, B., Shi, Z.-J., Yan, J.-Y., Li, J.-H., and Feng, S.-Q. (2017). Long non-coding RNA NONMMUG014387 promotes Schwann cell proliferation after peripheral nerve injury. Neural Regen. Res. 12, 2084-2091. doi: 10.4103/16735374.221168

Piñero, G., Berg, R., Andersen, N. D., Setton-Avruj, P., and Monje, P. V. (2017). Lithium reversibly inhibits Schwann cell proliferation and differentiation without inducing myelin loss. Mol. Neurobiol. 54, 8287-8307. doi: 10.1007/s12035-016-0262-z

Sabater, A. L., Andreu, E. J., García-Guzmán, M., López, T., Abizanda, G., Perez, V. L., et al. (2017). Combined PI3K/Akt and Smad2 activation promotes corneal endothelial cell proliferation. Invest. Ophthalmol. Vis. Sci. 58, 745-754. doi: 10.1167 /iovs. 16-20817

Tricaud, N. (2017). Myelinating Schwann cell polarity and mechanically-driven myelin sheath elongation. Front. Cell. Neurosci. 11:414. doi: 10.3389/fncel.2017. 00414

Wang, C., Lu, C.-F., Peng, J., Hu, C.-D., and Wang, Y. (2017). Roles of neural stem cells in the repair of peripheral nerve injury. Neural Regen. Res. 12, 2106-2112. doi: 10.4103/1673-5374.221171

Wang, L., Wang, T., Song, M., and Pan, J. (2014). Rho plays a key role in TGF- $\beta 1$ induced proliferation and cytoskeleton rearrangement of human periodontal ligament cells. Arch. Oral Biol. 59, 149-157. doi: 10.1016/j.archoralbio. 2013.11.004

Wen, J., Qian, C., Pan, M., Wang, X., Li, Y., Lu, Y., et al. (2017a). Lentivirusmediated RNA interference targeting RhoA slacks the migration, proliferation and myelin formation of Schwann cells. Mol. Neurobiol. 54, 1229-1239. doi: $10.1007 /$ s12035-016-9733-5

Wen, J., Tan, D., Li, L., and Guo, J. (2017b). Isolation and purification of Schwann cells from spinal nerves of neonatal rat. Bio-protocol 7:e2588. doi: 10.21769/bioprotoc. 2588

Wen, J., Tan, D., Li, L., Wang, X., Pan, M., and Guo, J. (2018). RhoA regulates Schwann cell differentiation through JNK pathway. Exp. Neurol. 308, 26-34. doi: 10.1016/j.expneurol.2018.06.013

Wong, K. M., Babetto, E., and Beirowski, B. (2017). Axon degeneration: make the Schwann cell great again. Neural Regen. Res. 12, 518-524. doi: 10.4103/16735374.205000

Wu, K.-C., Cheng, K.-S., Wang, Y.-W., Chen, Y.-F., Wong, K.-L., Su, T.-H., et al. (2017). Perturbation of Akt signaling, mitochondrial potential and ADP/ATP ratio in acidosis-challenged rat cortical astrocytes. J. Cell. Biochem. 118, 1108-1117. doi: 10.1002/jcb.25725

Wu, W., Liu, Y., and Wang, Y. (2016). Sam68 promotes Schwann cell proliferation by enhancing the PI3K/Akt pathway and acts on regeneration after sciatic nerve crush. Biochem. Biophys. Res. Commun. 473, 1045-1051. doi: 10.1016/j.bbrc. 2016.04.013 
Yang, Q., Wen, L., Meng, Z., and Chen, Y. (2018). Blockage of endoplasmic reticulum stress attenuates nilotinib-induced cardiotoxicity by inhibition of the Akt-GSK3ß-Nox4 signaling. Eur. J. Pharmacol. 822, 85-94. doi: 10.1016/j. ejphar.2018.01.011

Zhang, L.-L., Mu, G.-G., Ding, Q.-S., Li, Y.-X., Shi, Y.-B., Dai, J.-F., et al.. (2015). Phosphatase and tensin homolog (PTEN) represses colon cancer progression through inhibiting paxillin transcription via PI3K/AKT/NF-кB pathway*. J. Biol. Chem. 290, 15018-15029. doi: 10.1074/jbc.m115.641407

Zhao, Z., Li, X., and Li, Q. (2017). Curcumin accelerates the repair of sciatic nerve injury in rats through reducing Schwann cells apoptosis and promoting myelinization. Biomed. Pharmacother. 92, 1103-1110. doi: 10.1016/j.biopha. 2017.05.099

Zhou, J., Chen, J., and Yu, H. (2018). Targeting sphingosine kinase 2 by ABC294640 inhibits human skin squamous cell carcinoma cell growth. Biochem. Biophys. Res. Commun. 497, 535-542. doi: 10.1016/j.bbrc.2018.02.075
Zhou, Z., Peng, X., Chiang, P., Kim, J., Sun, X., Fink, D. J., et al. (2012). HSVmediated gene transfer of C3 transferase inhibits Rho to promote axonal regeneration. Exp. Neurol. 237, 126-133. doi: 10.1016/j.expneurol.2012.06.016

Conflict of Interest Statement: The authors declare that the research was conducted in the absence of any commercial or financial relationships that could be construed as a potential conflict of interest.

Copyright (c) 2018 Tan, Wen, Li, Wang, Qian, Pan, Lai, Deng, Hu, Zhang and Guo. This is an open-access article distributed under the terms of the Creative Commons Attribution License (CC BY). The use, distribution or reproduction in other forums is permitted, provided the original author(s) and the copyright owner(s) are credited and that the original publication in this journal is cited, in accordance with accepted academic practice. No use, distribution or reproduction is permitted which does not comply with these terms. 